\title{
Algebraic Number Precoded OFDM Transmission for Asynchronous Cooperative Multirelay Networks
}

\author{
Hua Jiang, ${ }^{1}$ Lizhen Shen, ${ }^{2}$ Hao Cheng, ${ }^{1}$ and Guoqing Liu ${ }^{1}$ \\ ${ }^{1}$ School of Sciences, Nanjing Tech University, Nanjing 211816, China \\ ${ }^{2}$ Biotechnology and Pharmaceutical Engineering, Nanjing Tech University, Nanjing 211816, China \\ Correspondence should be addressed to Guoqing Liu; gqliunj@163.com
}

Received 28 July 2014; Accepted 25 October 2014; Published 4 November 2014

Academic Editor: Jun-Juh Yan

Copyright (c) 2014 Hua Jiang et al. This is an open access article distributed under the Creative Commons Attribution License, which permits unrestricted use, distribution, and reproduction in any medium, provided the original work is properly cited.

\begin{abstract}
This paper proposes a space-time block coding (STBC) transmission scheme for asynchronous cooperative systems. By combination of rotated complex constellations and Hadamard transform, these constructed codes are capable of achieving full cooperative diversity with the analysis of the pairwise error probability (PEP). Due to the asynchronous characteristic of cooperative systems, orthogonal frequency division multiplexing (OFDM) technique with cyclic prefix (CP) is adopted for combating timing delays from relay nodes. The total transmit power across the entire network is fixed and appropriate power allocation can be implemented to optimize the network performance. The relay nodes do not require decoding and demodulation operation, resulting in a low complexity. Besides, there is no delay for forwarding the OFDM symbols to the destination node. At the destination node the received signals have the corresponding STBC structure on each subcarrier. In order to reduce the decoding complexity, the sphere decoder is implemented for fast data decoding. Bit error rate (BER) performance demonstrates the effectiveness of the proposed scheme.
\end{abstract}

\section{Introduction}

Recently, cooperative communication networks are known to have significant potential in increasing network capacity and transmission reliability. In recent years, cooperative networks have attracted substantial interest from the wireless networking and communications research [1-13]. The basic idea is that intermediate relay nodes act as a virtual distributed antenna array to assist the source node in transmitting its information to the destination node. The amplify-and-forward (AF) and decode-and-forward (DF) are the most famous schemes for cooperative systems [14].

Space-time block code (STBC) $[15,16]$ is an effective approach to achieve spatial diversity for cooperative transmissions. Since the relay nodes are in different locations and have different oscillators, there may exist timing errors. Therefore, it is difficult to design proper space-time coding schemes. The transmission schemes, which are based on orthogonal frequency division multiplexing (OFDM), are exploited for combating the loss of timing phase [2-5]. In [4], a simple Alamouti scheme is proposed to achieve cooperative diversity, where only a few simple operations such as time-reversion and complex conjugation are required at the relay nodes; the fast ML Alamouti decoding is used at the destination node. However, this scheme is only useful for the case of two relay nodes. In [6, 7], the clustered orthogonal space-time block code (OSTBC) schemes for four or more relay nodes are proposed by clustering the relay nodes. It is shown that limited performance improvement is achieved while the number of relay nodes increases.

In [17-21], a new multidimensional modulation scheme is proposed to increase the modulation diversity. It is feasible to achieve full modulation diversity by applying optimum rotation to signal constellation. The algebraic number theory is introduced in [22] to construct a multidimensional rotation matrix. By properly selecting the algebraic number field, a generator matrix that guarantees full modulation diversity and maximizes the minimum product distance for precoded information symbols can be obtained.

In this paper we study the diagonal algebraic space-time (DAST) block coding for asynchronous cooperative network. We consider the OFDM technique with enough cyclic prefix 
(CP) $[23,24]$ to combat the timing errors. The information symbols are precoded by a matrix multiplication at the source node. Throughout the proper operation at the relay node, the received signals hold the DAST block code structure on each subcarrier; hence it is convenient to decode data by using ML decoding or fast sphere decoder. It is assumed that the relay nodes do not have to know any information about the channels but the destination node knows all channel information through training. Therefore the relay nodes do not need to decode and demodulate signals received from the source node. Only a few simple signal processings are needed at the relay nodes.

This paper is organized as follows. In Section 2, the relay network model is described and a new transmission scheme is proposed. In Section 3, the algebraic number theory is introduced and the optimal rotation constellation scheme is presented. The PEP is analyzed with the optimal power allocation in Section 4. Section 5 contains the simulation results. Finally, the conclusions are given in Section 6.

Notation. For a vector or matrix $A, A^{T}, A^{H}$, and $\|A\|$ indicate the transpose, Hermitian, and Frobenius norm of $A$, respectively. $A \circ B$ denotes the Hadamard product of $A$ and $B$, that is, the componentwise product. * indicates the conjugate operation. $\circledast$ denotes the circular convolution. $\operatorname{diag}\left\{a_{1}, \ldots, a_{n}\right\}$ is a diagonal matrix with $a_{i}$ being its $i$ th diagonal entry. $E$ indicates the expectation and $P$ indicates the probability. Gaussian integer is a complex number whose real and imaginary parts are both integers.

\section{Relay Network Model and Cooperative Protocol}

Consider a relay network with one source node, one destination node, and $R\left(R=2^{q}, q=1,2, \ldots\right)$ relay nodes, as shown in Figure 1 . There is only one antenna at every node. We denote the average signal energy $E_{s}=1$ by a normalizd QAM constellation. OFDM technique is used to combat the time errors. It is assumed that the number of subcarriers is $N$ and the transmission delay of the signals from $i$ th relay node at the destination node is $\tau_{i}$, which is a multiple of $T_{s}$ with $T_{s}$ denoting the information symbol duration. At the source node the information bits are modulated into complex symbols by normalized QAM constellation. The consecutive $N R$ symbols are denoted by $s_{j, k}, j=1,2, \ldots, R$, $k=1,2, \ldots, N$. Then we let $s_{j}=\left[s_{j, 1}, s_{j, 2}, \ldots, s_{j, N}\right]$ represent the $j$ th block consisting of $N$ symbols.

Let $M$ be an $R \times R$ precoding matrix. We obtain the precoded symbols $\widehat{x}_{k}=\left[x_{1, k}, \ldots, x_{R, k}\right]^{T}=M \widehat{s}_{k}$, where $\widehat{s}_{k}=\left[s_{1, k}, \ldots, s_{R, k}\right]^{T}$. Then the new $R$ consecutive OFDM block is denoted by $x_{j}=\left[x_{j, 1}, x_{j, 2}, \ldots, x_{j, N}\right]$. After $N$-point IFFT operation and CP of length $\ell_{\mathrm{cp}}$ insertion, the OFDM symbols of length $N+\ell_{\mathrm{cp}}$ are transmitted to the destination node through the relay nodes. We assume that the knowledge of timing errors can be obtained at the destination node. It is emphasized that the CP length must be larger than the maximum time delay $\tau_{\max }$. Besides, we make the assumption that the channels between any two nodes are quasi-static flat

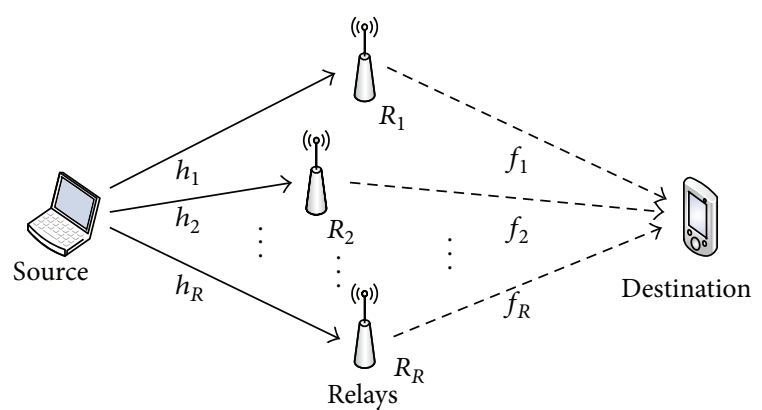

FIgURE 1: Relay network model.

fading. The fading coefficient from the source node to the $i$ th relay node is denoted by $h_{i}$, and the fading coefficient from $i$ th relay node to the destination node is denoted by $f_{i}$. These coefficients are independent and identically distributed (i.i.d.) complex Gaussian random variables with zero mean and unit variance.

Denote by $X_{1}, \ldots, X_{R}$ the $R$ consecutive OFDM symbols, where $X_{j}$ consists of $\operatorname{IFFT}\left(x_{j}\right)$ and the corresponding CP for $j=1, \ldots, R$. The $k$ th subcarrier output of $j$ th OFDM symbols in frequency domain can be represented by

$$
\operatorname{IFFT}\left(x_{j}\right)(k)=\frac{1}{\sqrt{N}} \sum_{u=1}^{N} x_{j, u} e^{2 \pi \sqrt{-1}(u-1)(k-1) / N} .
$$

The $1 / \sqrt{N}$ multiplier in terms of IFFT guarantees the power of signal symbols invariant after IFFT operation. We assume the channel coefficients remain unchanged during the transmission of $R$ OFDM symbols. At the $i$ th relay node in the $j$ th OFDM symbol duration the received signals are

$$
Y_{i, j}=\sqrt{P_{1}} X_{j} h_{i}+n_{i, j}
$$

where $P_{1}$ is the average transmit power at the source node and $n_{i, j}$ is the corresponding additive white Gaussian noise (AWGN) with zero mean and unit variance at the $j$ th relay node, in the $i$ th OFDM symbol duration. Note that due to the additive white Gaussian noise, the average power of received signal is $P_{1}+1$.

The relay nodes would simply process and transmit the received noisy signals. Only unary positive and negative operations are needed. For instance, in the case of $R=4$ the processed signal matrix is given by

$$
\bar{Y}=\lambda\left[\begin{array}{cccc}
Y_{1,1} & Y_{2,1} & Y_{3,1} & Y_{4,1} \\
Y_{1,2} & -Y_{2,2} & Y_{3,2} & -Y_{4,2} \\
Y_{1,3} & Y_{2,3} & -Y_{3,3} & -Y_{4,3} \\
Y_{1,4} & -Y_{2,4} & -Y_{3,4} & Y_{4,4}
\end{array}\right]
$$

We can find that it holds the structure similar to the Hadamard matrix. Apart from the orthogonal STBC scheme, which is required to switch the OFDM symbols and has to wait to start process and transmit until the next several OFDM symbols arrive at the relay nodes $[4,5]$, in our proposed scheme the relay nodes can process and broadcast the received signals immediately without waiting the other 
symbols arriving. As a result, the proposed scheme would not induce the transmission delay.

Let $\bar{Y}_{i, j}=\lambda S_{i, j} Y_{i, j}$ be the transmit signal from the $i$ th relay node, in the $j$ th OFDM symbol duration, where $S_{i, j}$ belonging to $\{+1,-1\}$ is the entry of Hadamard matrix $S$ of $R$ dimensions, and scalar $\lambda=\sqrt{P_{2} /\left(P_{1}+1\right)}$ guarantees the average transmit power is $P_{2}$ for one transmission at every relay node. At the destination node, the received signal in the $j$ th OFDM symbol duration can be written as

$$
\bar{Z}_{j}=\sum_{i=1}^{R}\left(\bar{Y}_{i, j} \circledast F_{i}\right) f_{i}+\bar{W}_{j},
$$

where $\bar{W}$ is the corresponding AWGN at the destination node and $F_{i}$ is an $N$ point vector whose $\left(\tau_{i}+1\right)$ th element is one and the others are zero. Time delays in the time domain are expressed by circular convolution with $F_{i}$. After CP removal and $N$-point FFT transformation, the received signals can be rewritten as

$$
Z_{j}=\lambda \sum_{i=1}^{R} S_{i, j}\left(\sqrt{P_{1}} x_{j} \circ f^{\tau_{i}} h_{i} f_{i}+N_{i, j} \circ f^{\tau_{i}} f_{i}\right)+W_{j},
$$

where $f^{\tau_{i}}=\left[1, e^{-2 \pi \tau_{i} \sqrt{-1} / N}, \ldots, e^{-2 \pi \tau_{i} \sqrt{-1}(N-1) / N}\right]^{T}$ with $f=$ $\left[1, e^{-2 \pi \sqrt{-1} / N}, \ldots, e^{-2 \pi \sqrt{-1}(N-1) / N}\right]^{T}$ meaning the phase change in frequency domain corresponding to the sample time delay $\tau_{i}$ in time domain, $N_{i, j}=\operatorname{FFT}\left(n_{i, j}\right)$, and $W_{j}=\operatorname{FFT}\left(\bar{W}_{j}\right)$. For every subcarrier $k, 1 \leq k \leq N$, we have

$$
\begin{aligned}
z_{k}= & \lambda \sqrt{P_{1}}\left(\operatorname{diag}\left(x_{1, k}, x_{2, k}, \ldots, x_{R, k}\right) S\right)\left(f_{k}^{\tau} \circ h \circ f\right) \\
& +\lambda\left(N_{k} \circ S\right)\left(f_{k}^{\tau} \circ f\right)+w_{k},
\end{aligned}
$$

where we have defined

$$
\begin{gathered}
z_{k}=\left[\begin{array}{c}
z_{1, k} \\
\vdots \\
z_{R, k}
\end{array}\right], \quad f_{k}^{\tau}=\left[\begin{array}{c}
f_{k}^{\tau_{1}} \\
\vdots \\
f_{k}^{\tau_{R}}
\end{array}\right], \\
h=\left[\begin{array}{c}
h_{1} \\
\vdots \\
h_{R}
\end{array}\right], \quad f=\left[\begin{array}{c}
f_{1} \\
\vdots \\
f_{R}
\end{array}\right], \\
w_{k}=\left[\begin{array}{c}
w_{1, k} \\
\vdots \\
w_{R, k}
\end{array}\right] .
\end{gathered}
$$

$N_{k}$ is an $R \times R$ matrix with the entries $\left\{N_{i, j, k}\right\}, 1 \leq i \leq R$, $1 \leq j \leq R$. The Hadamard transform is useful for reducing the high peak-to-average power ratio over different transmit antennas resulting in power amplification.

\section{Rotated Constellations Using Algebraic Number Theory}

In order to achieve full modulation diversity over the Rayleigh fading channel and Gaussian fading channel, the rotation of a multidimensional signal symbol vector is discussed in this section. The minimum product distance of the constellation considered is defined as

$$
d_{\min }=\min _{x=M\left(s-s^{\prime}\right), s \neq s^{\prime}} \prod_{j=1}^{R}\left|x_{j}\right|,
$$

where $s$ and $s^{\prime}$ belong to an $R$-dimensional constellation (QAM or PAM). The algebraic number theory was employed to construct a proper precoding rotation matrix $M$, which maximizes the minimum product distances in certain dimensions. The diversity order is the minimum Hamming distance between any two coordinate vectors of constellation points. It is emphasized that a rotation matrix constructed by algebraic number theory is a Vandermonde matrix, which can simplify the encoding and reduce the computational complexity in a similar way of fast Fourier transformation. Furthermore, this algebraic construction of rotations is useful for reduction of peak-to-mean envelope power ratio (PMEPR).

It can be considered that the algebraic constellation has full spatial diversity if the associated minimum product distance $d_{\min }$ is strictly positive; equivalently, the components of vectors $x=M s$ and $x^{\prime}=M s^{\prime}$ (with $s \neq s^{\prime}$ ) in the rotated constellation are all different. To construct a precoding matrix $M$ of dimension $R\left(R=2^{q}, q=1,2, \ldots\right)$ with full modulation diversity, we apply the canonical embedding to some totally complex cyclotomic number fields. The reader can refer to $[25,26]$ for more comprehensive details about cyclotomic number fields and canonical embedding.

The algebraic norm for real integer in number fields at first appears in solving problems such as the integer solutions of finding all $x^{n}+y^{n}=z^{n}$, for $n=2$, which is stated in the Fermat theorem. It is emphasized that $N(\alpha)$ is an integer and $N(\alpha)=0$ if and only if $\alpha=0$; hence $N(\alpha) \geq 1$ for $\alpha \neq 0$. The algebraic norm should be compared with the canonical embedding. With the application of the canonical embedding $\sigma_{i}$ to each element of basis $\left[1, \theta, \theta^{2}, \ldots, \theta^{N-1}\right]$ of $K$, the generator matrix is given by

$$
M=\frac{1}{\sqrt{R}}\left[\begin{array}{cccc}
1 & \theta_{1} & \cdots & \theta_{1}^{R-1} \\
1 & \theta_{2} & \cdots & \theta_{2}^{R-1} \\
\vdots & \vdots & \ddots & \vdots \\
1 & \theta_{R} & \cdots & \theta_{R}^{R-1}
\end{array}\right]=\left[\xi_{1}, \xi_{2}, \ldots, \xi_{R}\right]
$$

where $\xi_{k}$ is the corresponding column of $M$ for $k=$ $1,2, \ldots, R$. Due to the characteristic of Vandermonde matrix and $\theta_{u}-\theta_{v} \neq 0$ for $1 \leq u \neq v \leq R$, the matrix $M$ has full rank. This means that matrix $M$ is eligible to be a generator complex matrix for multidimensional rotated constellation. It is important to select the roots $\theta_{k}, k=$ $1,2, \ldots, R$ for generating full modulation diversity rotations and maximizing the minimum product distance. With proper $\theta_{k}$ the matrix $M$ becomes an orthogonal matrix; that is, $M^{H} M=I$. According to the property of algebraic norm, the minimum product distance is a nonzero integer. It is revealed that $d_{\min }$ is related to the special properties of algebraic number field when the full modulation diversity is obtained. In fact, the diversity product is 1 for the optimal cyclotomic rotation matrix no matter what the space-time code size and constellation size are, which have been proved in [26]. 
We consider any two columns $\xi_{u}$ and $\xi_{v}$ of $M, u, v=$ $1,2, \ldots, R$; without loss of generality, we assume $v<u$; then the complex inner product of $\xi_{u}$ and $\xi_{v}$ is

$$
\left\langle\xi_{u}, \xi_{v}\right\rangle=\frac{1}{R} \sum_{k=1}^{R}\left(\theta_{k}\right)^{u-1}\left(\theta_{k}^{*}\right)^{v-1}=\frac{1}{R} \sum_{k=1}^{R}\left(\theta_{k} \theta_{k}^{*}\right)^{v}\left(\theta_{k}\right)^{u-v} .
$$

The problem transforms to the $R-1$ power symmetric functions with $R$ complex roots, with the constraints of the properties of the minimal polynomial $\mu_{\theta}(x)$. Due to the fact that the complex roots $\theta_{k}$ are on the unit circle, we hold $\theta_{k} \theta_{k}^{*}=1$ and $M$ is an orthogonal matrix, so that we obtain

$$
\left\langle\xi_{u}, \xi_{v}\right\rangle=\frac{1}{R} \sum_{k=1}^{R}\left(\theta_{k}\right)^{m}=0, \quad m=1,2, \ldots, R-1 .
$$

We assume the minimal polynomial

$$
\mu_{\theta}(x)=\prod_{k=1}^{R}\left(x-\theta_{k}\right)=\sum_{m=0}^{R} a_{m} x^{m}
$$

where $a_{R}=1, a_{R-1}=\sum_{1 \leq k \leq R} \theta_{k}, a_{R-2}=\sum_{1 \leq u<v \leq R} \theta_{u} \theta_{v}, \ldots$, and $a_{0}=\theta_{1} \theta_{2} \cdots \theta_{R}$. Notice that it is an elementary symmetric polynomial. According to Newton's identities, we have

$$
a_{m}=\frac{1}{R-m} \sum_{j=1}^{R-m}\left((-1)^{j-1} a_{m+j} \sum_{k=1}^{R}\left(\theta_{k}\right)^{j}\right) \text {. }
$$

Since $\sum_{k=1}^{R}\left(\theta_{k}\right)^{m}=0$ for $m=1,2, \ldots, R-1$, we have $a_{k}=0$ for $k=1,2, \ldots, R-1$. Notice the coefficients of the minimal polynomial $\mu_{\theta}(x)$ are a Gaussian integer and $\theta_{1}^{R}=\theta^{R}=i$, where $i=\sqrt{-1}$, which yields

$$
\mu_{\theta}(x)=x^{R}-i .
$$

Using the above results it is easy to achieve the complex roots $\theta_{k}=\exp ((4 k-3) \pi i / 2 R)$ for $k=1, \ldots, R$.

\section{PEP Analysis and Optimal Power Allocation}

The optimum power allocation between the source node and the relay nodes is discussed in [10] to minimize the pairwise error probability (PEP), which is well known to be an important measure for performance analysis. In this section we focus on the high power regime and the upper bound of PEP and provide the theoretical bases for reducing the upper bound of PEP with the precoding matrix. At first, for simplicity's sake we rewrite (6) as follows:

$$
z_{k}=\lambda \sqrt{P_{1}} A M \widehat{s}_{k}+w_{k}^{\prime}
$$

where $A=\operatorname{diag}\left(\left(f_{k}^{\tau} \circ h \circ f\right)^{T} S\right)$ and $w_{k}^{\prime}=\lambda\left(N_{k} \circ\right.$ $S)\left(f_{k}^{\tau} \circ f\right)+w_{k}$. The expression (15) implies that equivalently the rotated constellation symbols are transmitted over the diagonal channel matrix in space and time. Since $\left|f_{k}^{\tau_{i}}\right|=$ 1 for any $i$, it is obvious to see that $w_{k}^{\prime}$ is an independent
Gaussian random vector, so that we can obtain $E\left(w_{k}^{\prime}\right)=0$ and $\operatorname{Var}\left(w_{k}^{\prime}\right)=\left(1+\lambda^{2} \sum_{i=1}^{R}\left|f_{i}\right|^{2}\right) I_{R}$. Assuming ideal channel state information (CSI), the maximum-likelihood (ML) decoding is implemented. The PEP of mistaking $\widehat{s}_{k}$ by $\widehat{s}_{k}^{\prime}$ is given by

$$
P\left(\widehat{s}_{k} \longrightarrow \widehat{s}_{k}^{\prime}\right)=\frac{1}{2} \underset{h, f}{E} e^{-P_{1} P_{2}\left(H^{H}\left(C^{\prime}-C\right)^{H}\left(C^{\prime}-C\right) H\right) / 4\left(1+P_{1}+P_{2} \sum_{i=1}^{R}\left|f_{i}\right|^{2}\right)},
$$

where $C$ and $C^{\prime}$ are corresponding code matrix of $\widehat{s}_{k}$ and $\hat{s}_{k}^{\prime}$, respectively. When $R$ goes to infinity, by the law of large numbers, it shows that $(1 / R) \sum_{i=1}^{R}\left|f_{i}\right|^{2}$ converges to 1 since the variance of $\sum_{i=1}^{R}\left|f_{i}\right|^{2}$ tends to zeros. Thus we have $\sum_{i=1}^{R}\left|f_{i}\right|^{2} \approx$ $R$. It is also implied that the fading has little effect when $R$ is large. We assume the total transmit power in the whole system is $P$ per symbol transmission; then we have $P=P_{1}+R P_{2}$ and

$$
\frac{P_{1} P_{2}}{2\left(1+P_{1}+P_{2} R\right)}=\frac{P_{1}\left(P-P_{1}\right)}{2 R(1+P)} \leq \frac{P^{2}}{8 R(1+P)} .
$$

It is not hard to see that the probability can be minimized when $P_{1}=P / 2$ and $P_{2}=P / 2 R$; that is, it is optimal to allocate half of the total power to the transmit node and the other half to the relay nodes. It is significant that every relay node only consumes a little amount of power to contribute to the transmission.

To obtain the upper bound of PEP we have to compute the expectation over $h, f$. Note that $H=F^{\prime} h$, where $F^{\prime}=$ $\operatorname{diag}\left(f_{k}^{\tau_{1}} f_{1}, \ldots, f_{k}^{\tau_{R}} f_{R}\right)$. Since $\left|f_{k}^{\tau_{i}}\right|=1$, we have $F=F^{\prime H} F^{\prime}=$ $\operatorname{diag}\left(\left|f_{1}\right|^{2}, \ldots,\left|f_{R}\right|^{2}\right)$. By using the tight upper bound of Gaussian tail function, we have the following approximate inequality:

$$
\begin{aligned}
P\left(\widehat{s}_{k} \longrightarrow \widehat{s}_{k}^{\prime}\right) & \\
& \leqslant \frac{1}{2} \underset{h, f}{E} e^{-\left(P^{2} / 16 R(1+P)\right) h^{H} F^{\prime H}\left(C^{\prime}-C\right)^{H}\left(C^{\prime}-C\right) F^{\prime} h} \\
& =\frac{1}{2} \underset{f}{E} \int \pi^{-R} \exp \left(-\frac{P_{1} P_{2}\left(h^{H} F^{\prime H} G F^{\prime} h\right)}{4\left(P_{1}+1\right) N_{0}}-h^{H} h\right) d h \\
& =\frac{1}{2} \underset{f}{E \operatorname{det}^{-1}}\left[I_{R}+\frac{P^{2}}{16 R(1+P)}\left(C^{\prime}-C\right)^{H}\left(C^{\prime}-C\right) F\right] .
\end{aligned}
$$

Due to the fact that $\left(C^{\prime}-C\right)^{H}\left(C^{\prime}-C\right)=S \operatorname{diag}\left(\mid \hat{x}_{k}^{(1)}-\right.$ $\left.\left.\widehat{x}_{k}^{\prime(1)}\right|^{2}, \ldots,\left|\widehat{x}_{k}^{(R)}-\widehat{x}_{k}^{\prime(R)}\right|^{2}\right) S$ and $S S=R I_{R}$, it is not hard to see $\operatorname{det}\left[\left(C^{\prime}-C\right)^{H}\left(C^{\prime}-C\right)\right] \geq R^{R} d_{\text {min }}^{2}=(R / E)^{R}$. With the knowledge that $\delta_{i}=\left|f_{i}\right|^{2}$ is $\chi^{2}$ distribution with 2 degrees of freedom and the corresponding probability density function is $f\left(\delta_{i}\right)=e^{-\delta_{i}}$, we obtain

$$
\begin{aligned}
P\left(\widehat{s}_{k} \longrightarrow \widehat{s}_{k}^{\prime}\right) & \lesssim \frac{1}{2} \prod_{i=1}^{R} \int_{0}^{\infty}\left(1+\frac{\delta_{i}}{\beta}\right)^{-1} e^{-\delta_{i}} d \delta_{i} \\
& =\frac{1}{2}[\beta \exp (\beta) E i(-\beta)]^{R},
\end{aligned}
$$


where

$$
\beta=\frac{16 E(1+P)}{P^{2}}, \quad E i(x)=\int_{-\infty}^{x} \frac{e^{t}}{t} d t
$$

is the exponential integral function. This upper bound is sufficient to derive the optimization criteria. On the high power regime of $\log P \gg 1$, we have

$$
\begin{gathered}
e^{16 E(1+P) / P^{2}}=1+O\left(\frac{1}{P}\right) \approx 1, \\
E i\left(\frac{16 E(1+P)}{P^{2}}\right)=-\log P+O(1) \approx-\log P ;
\end{gathered}
$$

thus

$$
\begin{aligned}
P\left(s \longrightarrow s^{\prime}\right) & \lessgtr \frac{1}{2}\left[\frac{16 E(1+P)}{P}\right]^{R}\left(\frac{\log P}{P}\right)^{R} \\
& \approx \frac{1}{2}(16 E)^{R} P^{-R(1-\log \log P / \log P)} .
\end{aligned}
$$

Therefore, the achieved diversity gain is $R(1-\log \log P / \log P)$, which is linear in the number of relay nodes. If $P$ increases greatly $(\log P \gg \log \log P)$, full diversity of $R$ is obtained, the same as the multiple-input multiple-output (MIMO) system with $R$ transmit antennas and one receive antenna.

\section{Simulation Results}

In this section, we present some simulated performance of DAST codes for wireless asynchronous cooperative networks. The MATLAB 8.0 sumulation tool was used for the simulation (on a Core i7-3770 $3.4 \mathrm{GHz}$ PC). The different values of the number of relays $R$ and total transmit power $P$ are considered. We assume that the length of OFDM subcarriers is $N=64$ and the length of cyclic prefix $\ell_{\text {cp }}=16$ with the total bandwidth of $10 \mathrm{MHz}$, and the OFDM symbol duration is $T_{s}=6.4 \mu \mathrm{s}$. To satisfy the conditions that the time delay must be less than the CP length, $\tau_{i}$ is randomly chosen from 0 to 15 with the uniform distribution, and $\tau_{1}$ is assumed to be 0 for the first relay node. The information symbol is modulated by normalized 4-QAM. We fix the total transmit power $P$, which is measured in decibel. Using the optimal power allocation strategy, the transmit power of the source node is $P / 2$ and the relay nodes share the other power. The average SNR at the destination node can be calculated to be $P^{2} / 4(P+1)$. When $P \gg 1$, the SNR becomes $P / 4$. At the destination node, the ML decoding of the DAST block code can be implemented by the sphere decoder to obtain almost the same performance at a moderate complexity. The performances of the unrotated constellation for the relay network and the DAST block code for the multiple-input multiple-output (MIMO) system are given. For the sake of comparison, the performances of Alamouti code scheme [4] are also shown.

In Figure 2, we show the decoding performance of the network system equipped with two relay nodes. Assume all the systems have the same total transmit power and all the nodes in the relay network systems have the same power

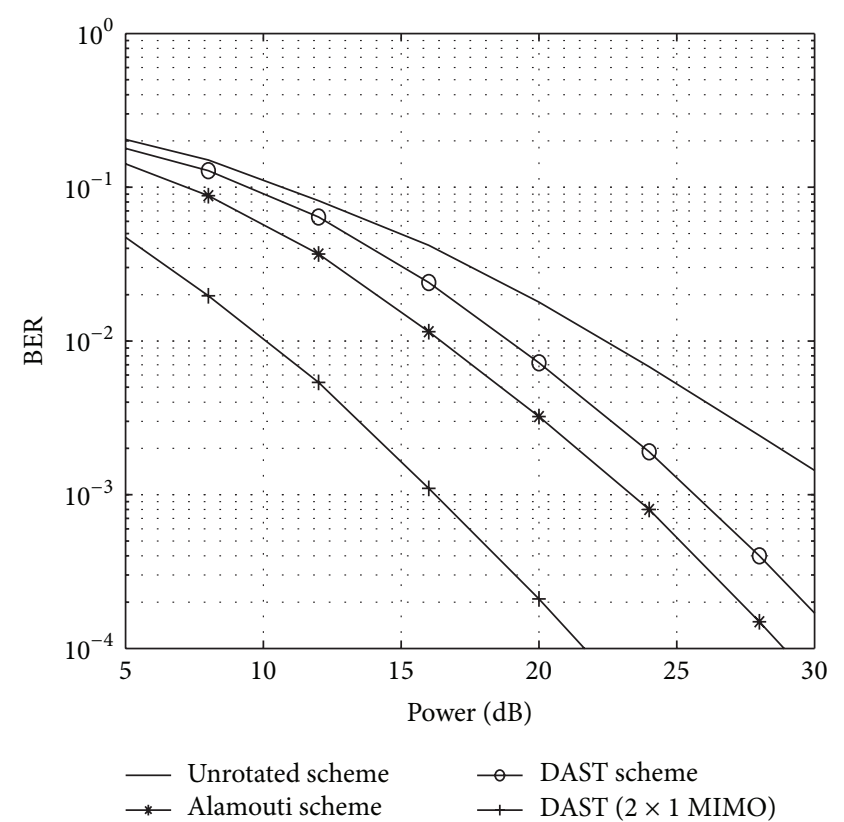

FIGURE 2: BER comparison of relay network with $R=2$.

constraint. As expected, we can see the performance for unrotated constellation is poor. For $R=2$, since the Alamouti scheme is the unique complex orthogonal design at a transmission rate of 1 , it seems hard for the DAST scheme to outperform it. However, there are some drawbacks for Alamouti scheme such that the relay nodes have to stack every two OFDM symbols and then exchange the transmit order of the two OFDM symbols, which leads to one OFDM symbol time slot delay. The advantage of DAST scheme will be immediate and significant when more relay nodes are employed.

Figures 3(a) and 3(b) show the decoding performance of the four-relay network systems and the MIMO system with 4 transmit antennas and 1 receive antenna. Figure 3(a) shows the BER performances with respect to the total transmit power. Figure 3(b) shows the BER performances with respect to the receive SNR. In the MIMO system the receive SNR is assumed to be $P$. We observe that the slopes of the BER curves of the DAST scheme approache the slopes of the BER of the DAST MIMO systems when the total transmit power $P$ or the receive SNR increases, which indicates that the new scheme can achieve diversity degree of 4 . We can see that the distance between the curves of the MIMO system and DAST relay system is large because the transmit antennas can fully cooperate in MIMO system. However, at the same receive SNR, the gap of the two curves is diminishing, which can be seen clearly in Figure 3(b). Since OSTBC design cannot achieve full transmission rate for more than two antennas, it is not applicable for the system with more than 2 relay nodes. Therefore, the cluster Alamouti code method [5] with rate $1 / 2$ is used for the network with 4 relay nodes. 16QAM is considered to maintain the same transmission rate. We can see that DAST scheme has a gain of about $3 \mathrm{~dB}$ at $10^{-6}$ in 


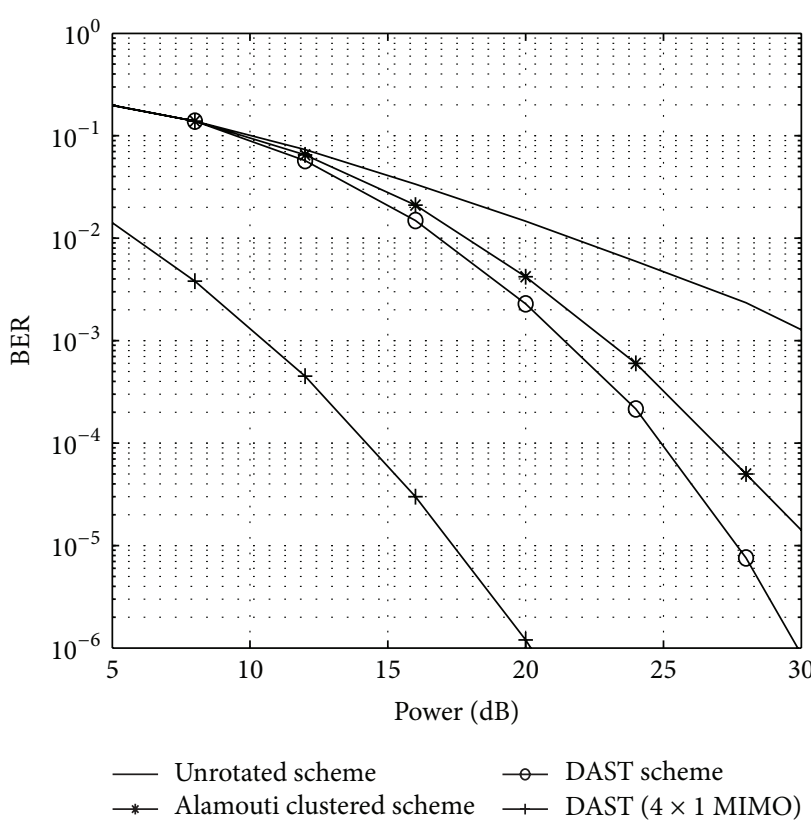

(a) The same total transmit power

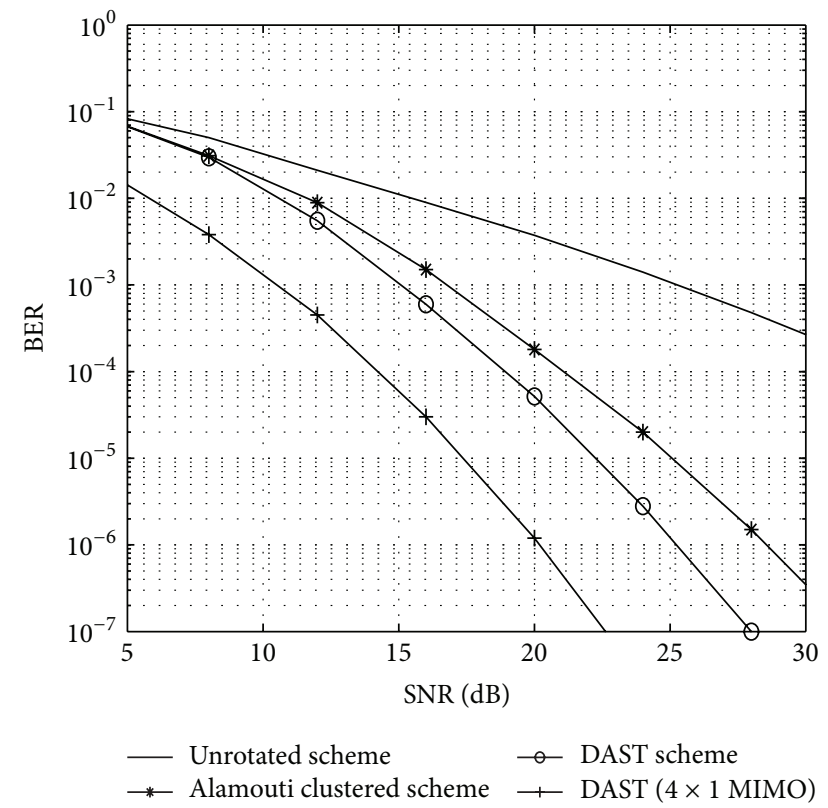

(b) The same receive SNR

FIGURE 3: BER comparison of relay network with $R=4$.

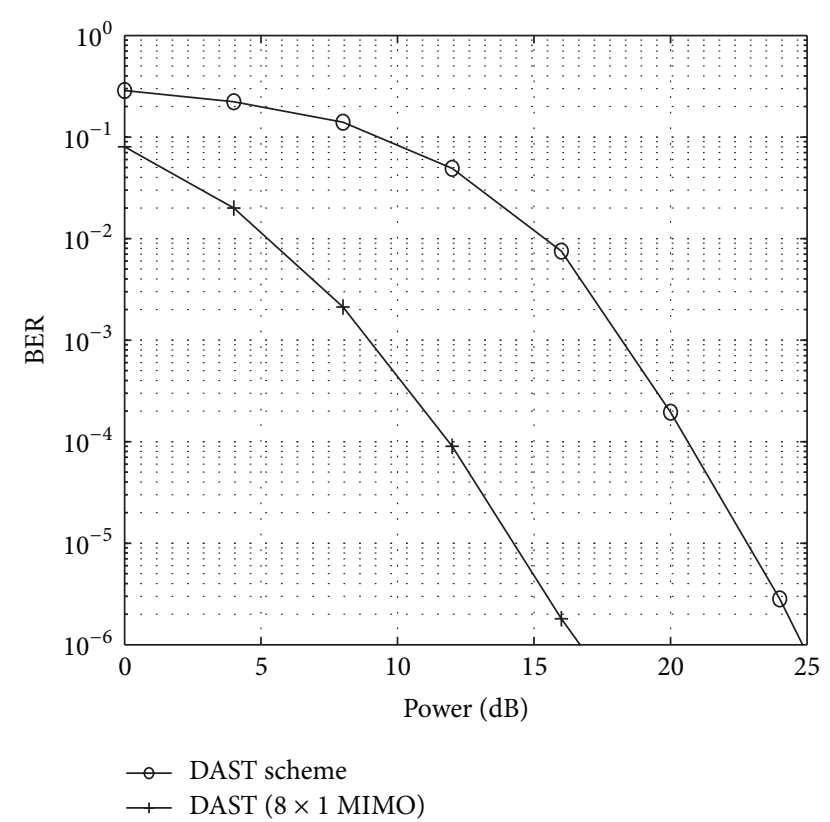

FIGURE 4: BER performance with $R=8$.

Figure 3(b). It should be noted that this gain will be enhanced when increasing the size of the constellation. In addition, the cluster Alamouti code scheme has one OFDM symbol time delay to perform signal processing at the relay nodes.

Finally, the example for $R=8$ is given to show the BER performance of the DAST scheme and the MIMO system with respect to the total transmit power in Figure 4. It also achieves a diversity order similar to that of DAST MIMO systems. When more relay nodes are utilized, the curves descend much faster at the high SNR regime. This implies the better BER performance is achieved.

\section{Conclusions}

In this paper, we propose the use of DAST block codes for asynchronous cooperative relay networks. OFDM technique is implemented at the source node, and only +/- operations are required at the relay nodes, without decoding and transmission delay. By using this method the received signal symbols at the destination node hold the STBC structure after removing $\mathrm{CP}$ and IFFT operation. This structure is useful for decoding. It can be considered efficient to adopt fast decoding algorithms such as sphere decoder to maintain the ML decoding performance in a polynomial time. It is not required with the transmitted signal symbols and channel information at the relay nodes. We analyze the PEP and observe that the proposed scheme is capable of achieving full spatial diversity for high total transmit power. Simulation results on the DAST scheme are demonstrated, which verifies our results.

For possible future works, it would be interesting to investigate further of imperfect CSI. In the existing works, the channels are assumed to be perfectly estimated. However, the channel estimation errors are inevitable in practice and affect the BER performance. Therefore, the investigation of impact of imperfect CSI on the proposed schemes would be our further work. Moreover, the symbol time offset and carrier frequency offset in OFDM technique can also be explored. 


\section{Conflict of Interests}

The authors declare that there is no conflict of interests regarding the publication of this paper.

\section{Acknowledgment}

This work was supported in part by the Natural Science Foundation of Jiangsu Province under Grant no. BK2011793.

\section{References}

[1] S. Wei, D. L. Goeckel, and M. C. Valenti, "Asynchronous cooperative diversity," IEEE Transactions on Wireless Communications, vol. 5, no. 6, pp. 1547-1557, 2006.

[2] H. Wang, Q. Yin, and X.-G. Xia, "Full diversity space-frequency codes for frequency asynchronous cooperative relay networks with linear receivers," IEEE Transactions on Communications, vol. 59, no. 1, pp. 236-247, 2011.

[3] X. Li, F. Ng, and T. Han, "Carrier frequency offset mitigation in asynchronous cooperative OFDM transmissions," IEEE Transactions on Signal Processing, vol. 56, no. 2, pp. 675-685, 2008.

[4] Z. Li and X.-G. Xia, "A simple Alamouti space-time transmission scheme for asynchronous cooperative systems," IEEE Signal Processing Letters, vol. 14, no. 11, pp. 804-807, 2007.

[5] Z. Li, X.-G. Xia, and M. H. Lee, "A simple orthogonal spacetime coding scheme for asynchronous cooperative systems for frequency selective fading channels," IEEE Transactions on Communications, vol. 58, no. 8, pp. 2219-2224, 2010.

[6] Z. Li, X.-G. Xia, and B. Li, "Achieving full diversity and fast ML decoding via simple analog network coding for asynchronous two-way relay networks," IEEE Transactions on Communications, vol. 57, no. 12, pp. 3672-3681, 2009.

[7] X. Guo and N.-G. Xia, "A distributed space-time coding in asynchronous wireless relay networks," IEEE Transactions on Wireless Communications, vol. 7, no. 5, pp. 1812-1816, 2008.

[8] S. Talwar, Y. Jing, and S. Shahbazpanahi, "Joint relay selection and power allocation for two-way relay networks," IEEE Signal Processing Letters, vol. 18, no. 2, pp. 91-94, 2011.

[9] Y. Jing and H. Jafarkhani, "Relay power allocation in distributed space-time coded networks with channel statistical information," IEEE Transactions on Wireless Communications, vol. 10, no. 2, pp. 443-449, 2011.

[10] Y. Jing and B. Hassibi, "Distributed space-time coding in wireless relay networks," IEEE Transactions on Wireless Communications, vol. 5, no. 12, pp. 3524-3536, 2006.

[11] B. Sirkeci-Mergen and A. Scaglione, "Randomized spacetime coding for distributed cooperative communication," IEEE Transactions on Signal Processing, vol. 55, no. 10, pp. 5003-5017, 2007.

[12] F. Oggier and B. Hassibi, "Cyclic distributed space-time codes for wireless relay networks with no channel information," IEEE Transactions on Information Theory, vol. 56, no. 1, pp. 250-265, 2010.

[13] Y. Jing, "Combination of MRC and distributed space-time coding in networks with multiple-antenna relays," IEEE Transactions on Wireless Communications, vol. 9, no. 8, pp. 2550-2559, 2010.

[14] R. U. Nabar, H. Bölcskei, and F. W. Kneubühler, "Fading relay channels: Performance limits and space-time signal design,"
IEEE Journal on Selected Areas in Communications, vol. 22, no. 6, pp. 1099-1109, 2004.

[15] S. M. Alamouti, "A simple transmit diversity technique for wireless communications," IEEE Journal on Selected Areas in Communications, vol. 16, no. 8, pp. 1451-1458, 1998.

[16] V. Tarokh, H. Jafarkhani, and A. R. Calderbank, "Spacetime block coding for wireless communications: performance results," IEEE Journal on Selected Areas in Communications, vol. 17, no. 3, pp. 451-460, 1999.

[17] H. El Gamal and M. O. Damen, "Universal space-time coding," IEEE Transactions on Information Theory, vol. 49, no. 5, pp. 1097-1119, 2003.

[18] X. Giraud, E. Boutillon, and J. Belfiore, "Algebraic tools to build modulation schemes for fading channels," IEEE Transactions on Information Theory, vol. 43, no. 3, pp. 938-952, 1997.

[19] P. Dayal and M. K. Varanasi, "An algebraic family of complex lattices for fading channels with application to space-time codes," IEEE Transactions on Information Theory, vol. 51, no. 12, pp. 4184-4202, 2005.

[20] B. A. Sethuraman, B. S. Rajan, and V. Shashidhar, "Full-diversity, high-rate space-time block codes from division algebras," IEEE Transactions on Information Theory, vol. 49, no. 10, pp. 25962616, 2003.

[21] M. O. Damen, K. Abed-Meraim, and J.-C. Belfiore, "Diagonal algebraic space-time block codes," IEEE Transactions on Information Theory, vol. 48, no. 3, pp. 628-636, 2002.

[22] J. Boutros and E. Viterbo, "Signal space diversity: a power- and bandwidth-efficient diversity technique for the Rayleigh fading channel," IEEE Transactions on Information Theory, vol. 44, no. 4, pp. 1453-1467, 1998.

[23] H. Abdzadeh-Ziabari, M. G. Shayesteh, and M. Manaffar, "An improved timing estimation method for OFDM systems," IEEE Transactions on Consumer Electronics, vol. 56, no. 4, pp. 2098 2105, 2010.

[24] Y. Jie, C. Lei, L. Quan, and C. De, "A modified selected mapping technique to reduce the peak-to-average power ratio of OFDM signal," IEEE Transactions on Consumer Electronics, vol. 53, no. 3, pp. 846-851, 2007.

[25] J. Boutros, E. Viterbo, C. Rastello, and J.-C. Belfiore, "Good lattice constellations for both rayleig $\mathrm{h}$ fading and gaussian channels," IEEE Transactions on Information Theory, vol. 42, no. 2, pp. 502-518, 1996.

[26] G. Wang and X.-G. Xia, "On optimal multilayer cyclotomic space-time code designs," IEEE Transactions on Information Theory, vol. 51, no. 3, pp. 1102-1135, 2005. 


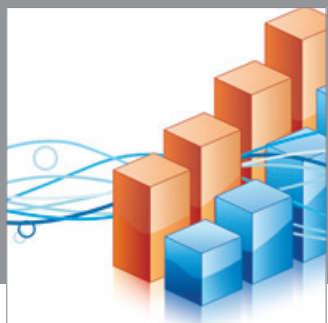

Advances in

Operations Research

mansans

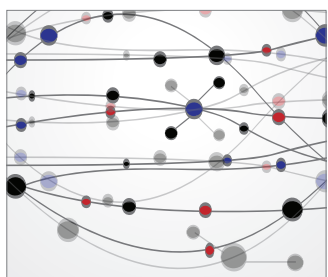

The Scientific World Journal
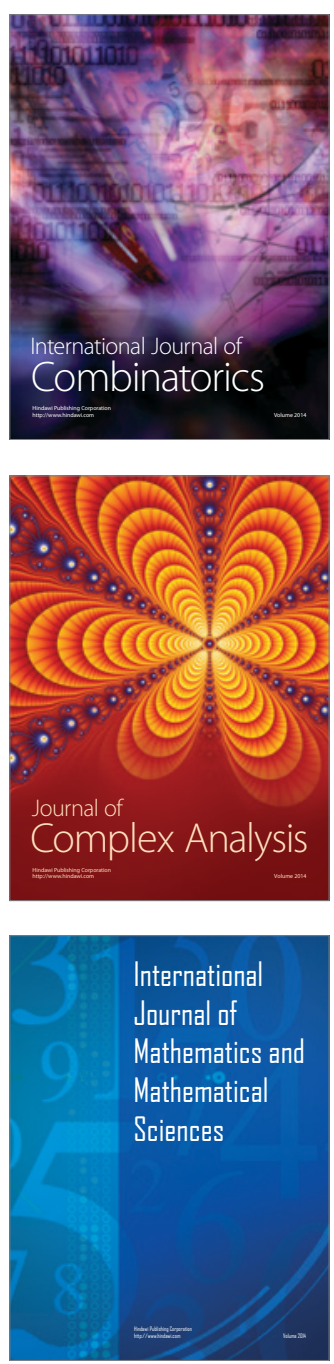
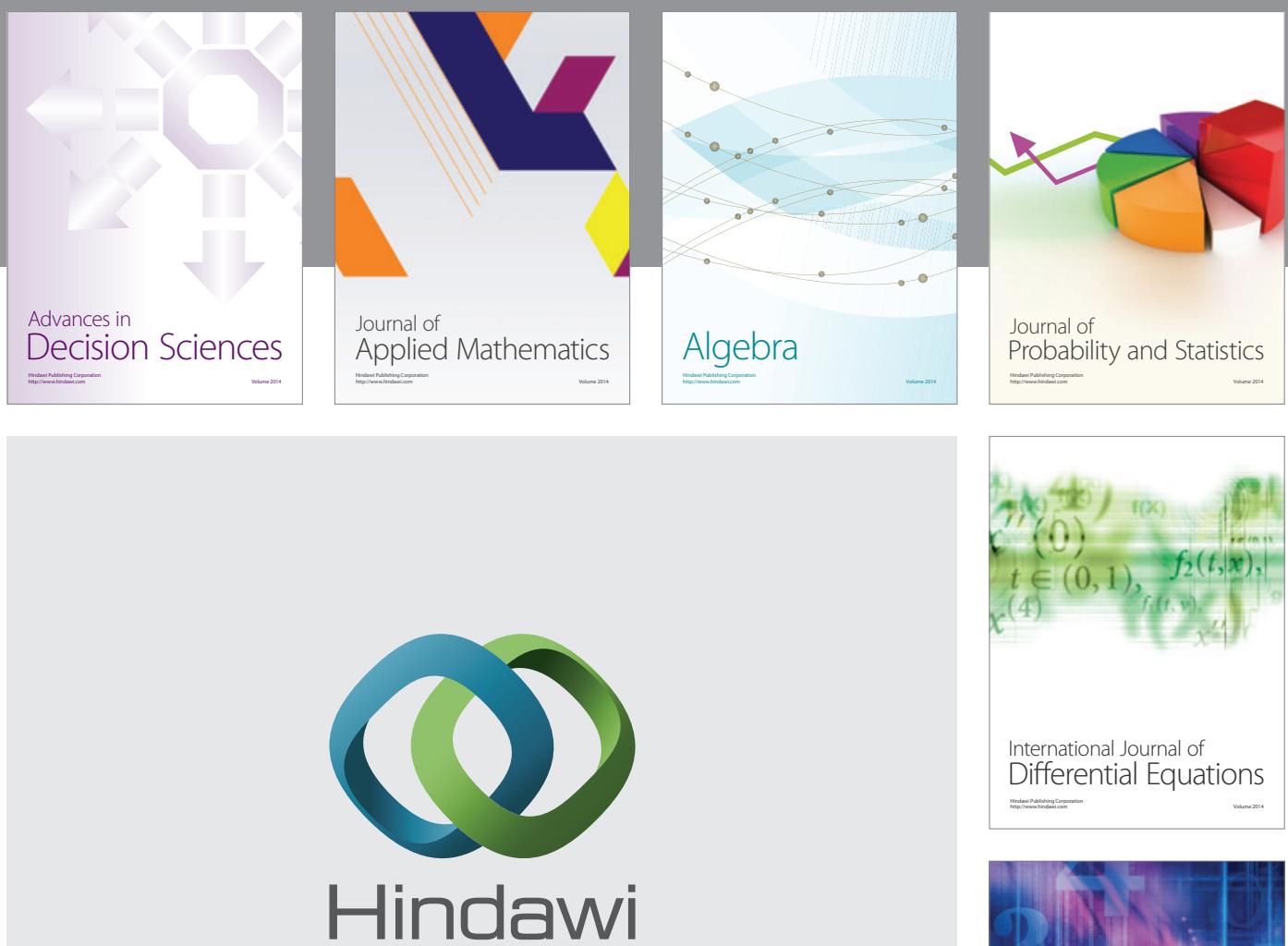

Submit your manuscripts at http://www.hindawi.com
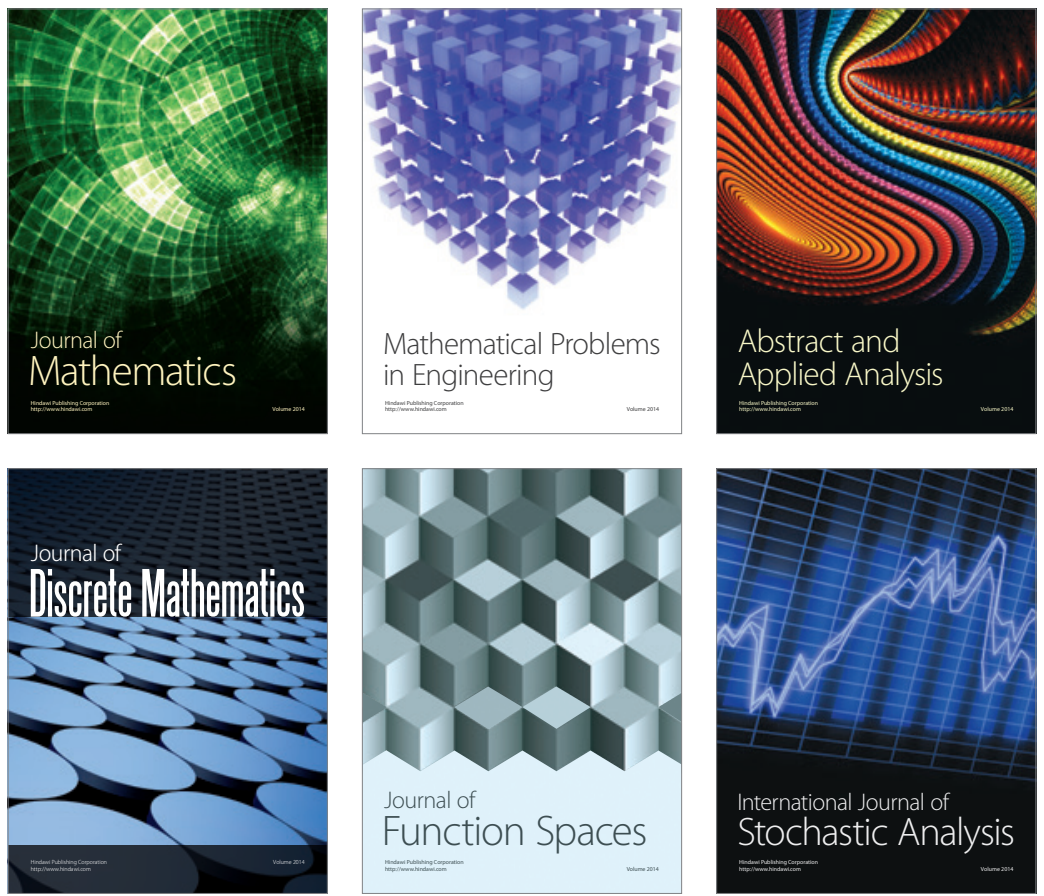

Journal of

Function Spaces

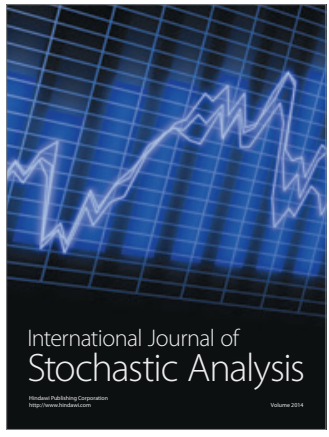

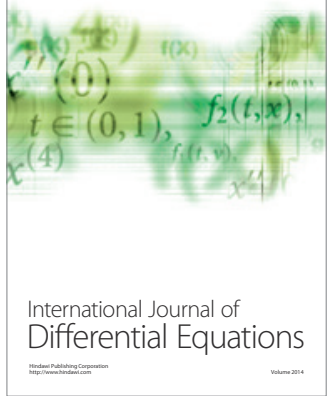
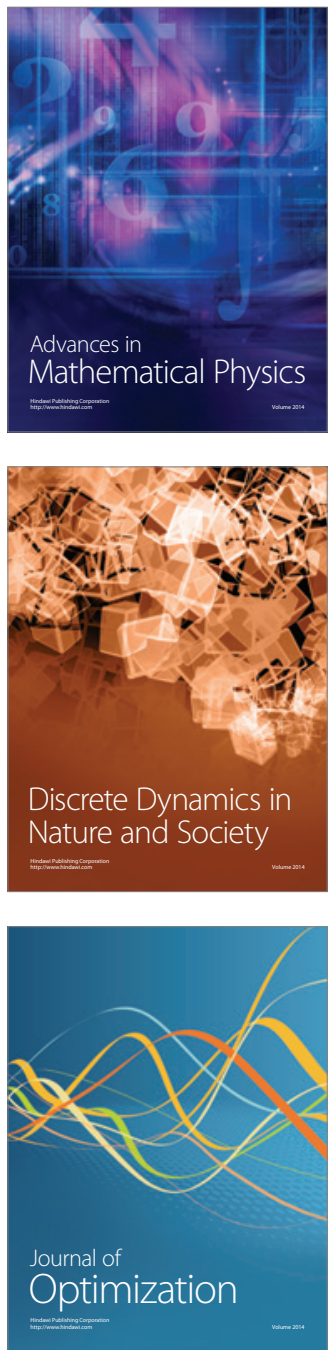